\title{
Mechanisms of deformable nanovesicles based on insulin-phospholipid complex for enhancing buccal delivery of insulin
}

This article was published in the following Dove Press journal: International Journal of Nanomedicine

\author{
You $X u^{1,2}$ \\ Xing Zhang ${ }^{1,2}$ \\ Yun Zhang ${ }^{1,2}$ \\ Jun Ye ${ }^{1,2}$ \\ Hong-Liang Wang ${ }^{1,2}$ \\ Xuejun $X^{1}{ }^{1,2}$ \\ Yuling Liu ${ }^{1,2}$ \\ 'State Key Laboratory of Bioactive \\ Substance and Function of Natural \\ Medicines, Institute of Materia \\ Medica, Chinese Academy of Medical \\ Sciences \& Peking Union Medical \\ College, Beijing 100050, China; \\ ${ }^{2}$ Beijing Key laboratory of Drug \\ Delivery Technology and Novel \\ Formulation, Institute of Materia \\ Medica, Chinese Academy of Medical \\ Sciences \& Peking Union Medical \\ College, Beijing 100050, China
}

Correspondence: Xuejun Xia; Yuling Liu Institute of Materia Medica, Chinese Academy of Medical Sciences \& Peking Union Medical College, I Xian Nong Tan Street, Beijing 100050, China

Tel +861083160332

Fax +861063159373

Email xjxia@imm.ac.cn;

ylliu@imm.ac.cn
Background: Non-injectable delivery of peptides and proteins are not feasible due to its large molecular, high hydrophilic and gastrointestinal degradation. Therefore, proposing a new method to solve this problem is a burning issue.

Purpose: The objective of this study was to propose a novel protein delivery strategy to vanquish the poor efficacy of buccal mucosa delivery systems for protein delivery and then investigate the detailed mechanisms of the enhanced buccal delivery of protein, using insulin as a model drug. Materials and methods: Insulin-phospholipid complex combined with deformable nanovesicles (IPC-DNVs) were prepared, using deformable nanovesicles based on insulin (INS-DNVs) and conventional nanovesicles based on insulin-phospholipid complex (IPC-NVs) as references. Besides, their physicochemical characterization, in vitro transport behavior, in vivo bioactivity and hypoglycemic effect were systematically characterized and compared. Finally, we evaluated the in vivo safety of IPC-DNVs.

Results: First, IPC-DNVs increased insulin permeability through deposition of the IPC and deformability of the DNVs, which was revealed by an in vitro mucosal permeation study. Second, DNVs could act as a drug carrier and penetrate the mucosa to reach the receiver medium as intact nanovesicles, which was supported by the observation of intact nanovesicles in the receiver medium through transmission electron microscopy (TEM). Third, IPC-DNVs exhibited both transcellular and paracellular transport in the form of IPC and DNVs, respectively, which was proved by confocal laser scanning microscopy (CLSM). Unlike the other two formulations, IPC-DNVs exhibited a sustained mild hypoglycemic effect, with a relative bioavailability (Fp) of $15.53 \%$ (3.09\% and $1.96 \%$ for INS-DNVs and IPC-NVs, respectively). Furthermore, buccal administration of IPC-DNVs resulted in no visible mucosal irritation to the buccal mucosa.

Conclusion: Our work reveals the mechanisms underlying the enhanced buccal delivery of IPC-DNVs: the DNVs facilitate penetration through the main barrier, and the deposition of IPC enhances buccal absorption. Our results and proposed mechanisms could be an important reference to understand other nanocarriers based on protein (peptide)-phospholipid complexes that penetrate the mucosa and provide a theoretical basis for the future development of buccal delivery systems for insulin.

Keywords: diabetes, hypoglycemic effect, mucosal permeation, absorption, safety

\section{Introduction}

Since the discovery of a phospholipid complex enhancing the bioavailability of poorly soluble drugs in drug delivery studies, the phospholipid complex technique has been extensively investigated to achieve good efficacy. ${ }^{1-3,8}$ In recent years, various phospholipid complex-based formulations such as Doxil ${ }^{\circledR},{ }^{4}$ Cleviprex $^{\circledR}, 5$ Valium ${ }^{\circledR}, 6$ and Silybin Phytosome ${ }^{\mathrm{TM}}{ }^{7}$ have been used clinically with good results. Among them, the formulations 
combing a phospholipid complex with therapeutic agents of high molecular weight or size (such as proteins, peptides, nucleic acids, and anticarcinogens) are particularly attractive because such strategies could help overcome the difficulty of directly encapsulating these therapeutic agents into carriers, ${ }^{8,9}$ thereby forming an amphiphilic complex and achieving a high drug-loading capacity and long-term stability. ${ }^{10,11}$ However, in non-injection routes, these phospholipid complex-based formulations have poor efficacy due to the low permeability of the skin or mucosa. ${ }^{12,13}$ In such cases, the phospholipid complex cannot penetrate the barrier, markedly limiting the efficacy of the drug. Thus, a novel delivery strategy with high mucosal permeability is highly desirable.

Recently, efforts have been devoted to develop noninjectable dosage forms of proteins and peptides, especially buccal mucosal delivery systems. ${ }^{14-16}$ Among them, deformable nanovesicles (DNVs), which were introduced by Cevc and Blume, ${ }^{17}$ markedly enhanced the membrane permeability for proteins and peptides. ${ }^{18}$ Inspired by this potential delivery platform, in this study, we report a novel protein delivery strategy that combines a phospholipid complex with DNVs, using insulin as a model drug (IPC-DNVs) for buccal absorption. In addition, we determined the transport mechanisms of IPC-DNVs using in vitro porcine buccal mucosa and in vivo rabbit model. To elucidate the mechanisms, we prepared IPC-DNVs, as well as DNVs based on insulin (INS-DNVs) and conventional nanovesicles based on insulin-phospholipid complex (IPC-NVs) as references. An in vitro mucosal permeation experiment was conducted to evaluate the penetration behavior of nanovesicles and to determine whether IPC-DNVs increase the absorption rate constant (flux), apparent permeability coefficient $\left(P_{\text {app }}\right)$, and deposition of insulin in the buccal mucosa. Transmission electron microscopy (TEM) was used to investigate whether DNVs could penetrate the mucosa in an intact form. Furthermore, confocal laser scanning microscopy (CLSM) was used to investigate the membrane transport of IPC-DNVs. An in vivo hypoglycemic study confirmed the mechanisms and demonstrated the importance of deformability and phospholipid complex in the buccal delivery of insulin. Finally, the in vivo safety was determined by acute irritation studies. Our results reveal the mechanisms underlying the enhanced buccal delivery of IPC-DNVs: the IPC acted as a penetration enhancer by fusing with the cell membrane, which enhanced penetration via transcellular transport, and the DNVs acted as a drug carrier system by penetrating the mucosa and reaching the receiver medium as intact nanovesicles via paracellular transport. Our mechanisms provide a reference to understand other protein (peptide)-phospholipid complex-based nanocarriers that penetrate the mucosa, which would greatly contribute to the development of non-injectable delivery systems.

\section{Materials and methods Materials}

Recombinant human insulin was purchased from Dongbao Enterprise Group Co., Ltd (Tonghua, Jilin, People's Republic of China). Lecithin (70\% phosphatidylcholine/30\% phosphatidylethanolamine, lipid phospholipid) was obtained from Shanghai Toshisun Biology and Technology Co., Ltd (Shanghai, People's Republic of China). Insulin-fluorescein isothiocyanate (FITC) was purchased from Meilun Biotechnology (Dalian, People's Republic of China). Rhodamine was purchased from Avanti (Alabaster, AL, USA). Other chemicals and solvents were of analytical or chromatography grade.

\section{Animals}

Male big-ear Japanese rabbits were purchased from Beijing Huafukang Bioscience Co., Inc. and were raised at the Institute of Material Medical, Chinese Academy of Medical Sciences and Peking Union Medical College (Beijing, People's Republic of China). The experiments were performed with the approval of the Laboratory Animal Care and Use Committee of Peking Union Medical College. All animal experiments were performed in accordance with the guidelines of laboratory animals - guideline for ethical review of animal welfare (GB_T 35892-2018) for the welfare of the animals (People's Republic of China).

\section{Preparation of nanovesicles}

Three types of nanovesicles were prepared using thin-film hydration method (Figure 1). For IPC-DNVs, the IPC was prepared by solvent evaporation method, as described in our previous study. ${ }^{19}$ Briefly, insulin (10 mg) and lipid (100 mg) were independently dissolved in $0.1 \%$ trifluoroacetic acidmethanol $(1 \mathrm{~mL})$ and dichloromethane $\left(9 \mathrm{~mL}, \mathrm{CH}_{2} \mathrm{CL}_{2}\right)$, respectively. After mixing for 10 minutes, the organic solvents were removed by rotary evaporation under vacuum to form IPC. The IPC was placed on a vacuum pump for 2 hours to remove the residual organic solvent. For IPC-DNVs, an appropriate amount of IPC was prepared as above; lipid and Tween 20 were dissolved in dichloromethane to form a clear solution. The organic solvent was evaporated at $37^{\circ} \mathrm{C}$ using a rotary evaporator to form a beehive film. The lipid films were hydrated with sodium deoxycholate (SDC)-PBS ( $\mathrm{pH} 7.4$ ) solution by rotation at $150 \mathrm{rpm}$ at $37^{\circ} \mathrm{C}$ for 30 minutes. To prepare small vesicles, the suspension was sonicated with cooling four times for 1 minute each. The sonicated vesicles were homogenized by manual extrusion through $0.22 \mu \mathrm{m}$ polycarbonate 
A

IPC-DNVs and IPC-NVs
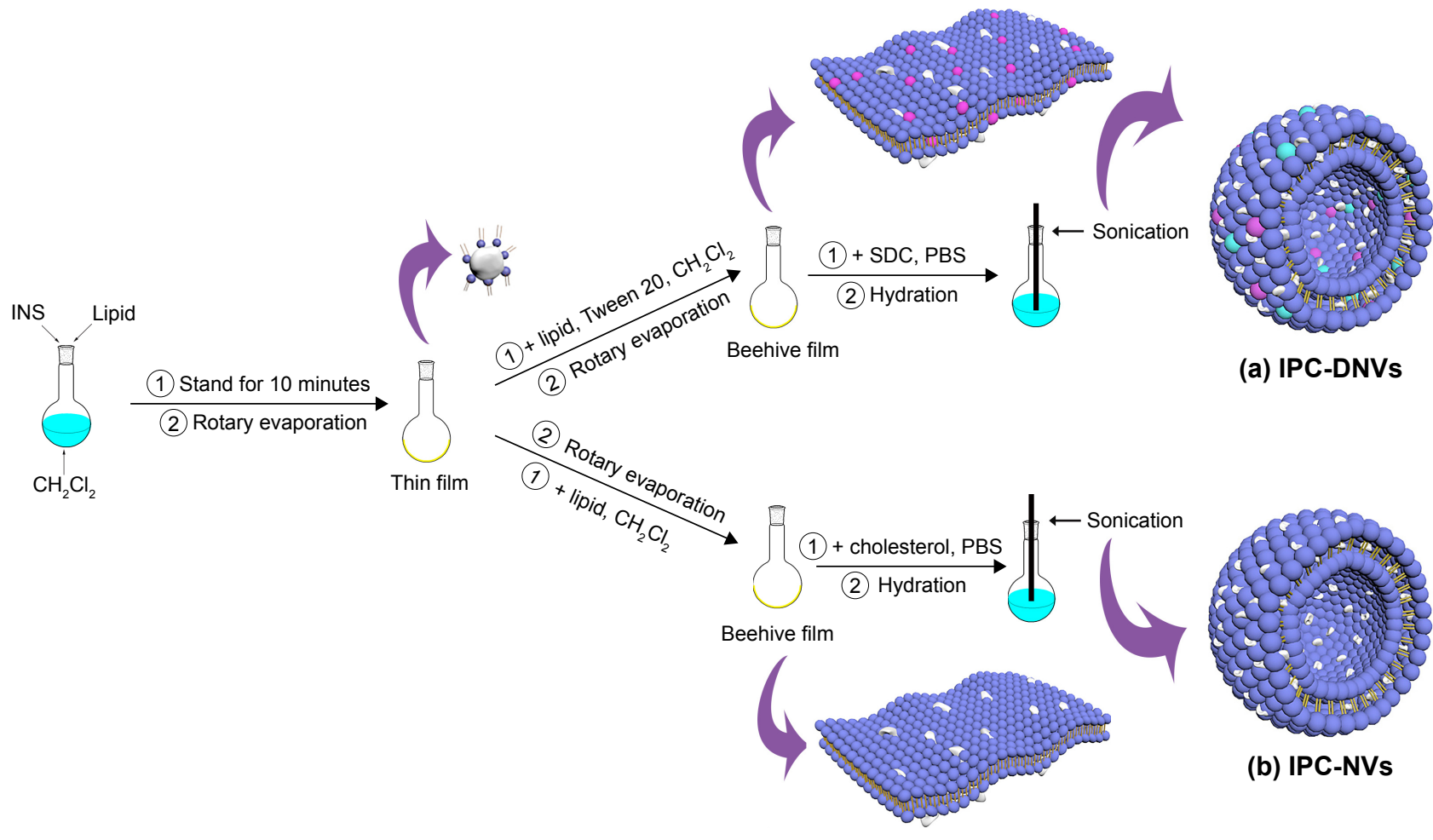

(a) IPC-DNVs

B

INS-DNVs

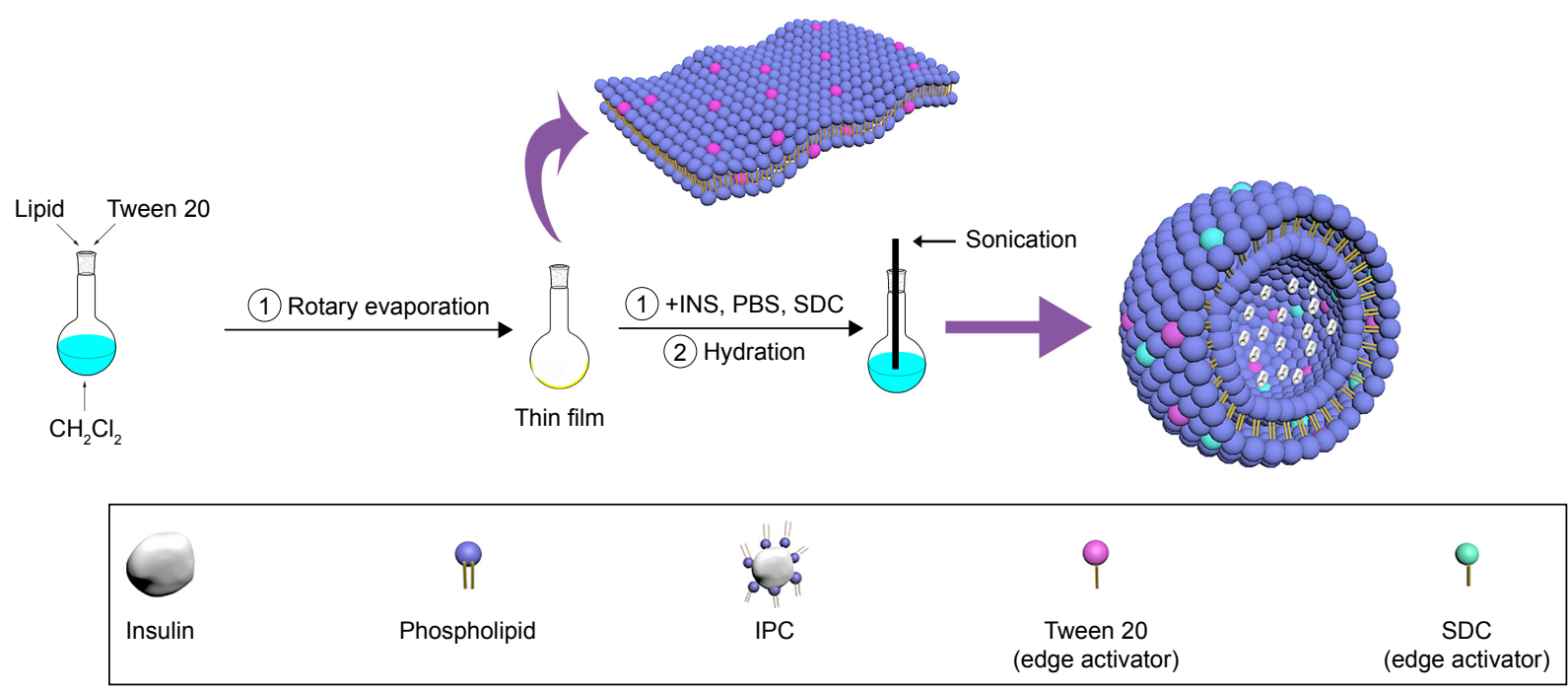

Figure I Schematic representation of the preparation process of (A) IPC-DNVs and IPC-NVs and (B) INS-DNVs.

Notes: For (A), INS dissolved in 0.1\% TFA-methanol, (a) IPC-DNVs, (b) IPC-NVs; for (B), INS dissolved in $0.1 \%$ TFA- $\mathrm{H}_{2} \mathrm{O}$.

Abbreviations: DNVs, deformable nanovesicles; INS, insulin; IPC, insulin-phospholipid complex; NVs, conventional nanovesicles; SDC, sodium deoxycholate; TFA, trifluoroacetic acid.

membranes. To prepare INS-DNVs, insulin (dissolved in $0.1 \%$ trifluoroacetic acid- $\mathrm{H}_{2} \mathrm{O}$ ) was added to SDC-PBS solution, and the other steps were the same as those for IPC-DNVs. For IPCNVs, Tween 20 and SDC were replaced with cholesterol to obtain the conventional nanovesicles. The final concentration of insulin in the nanovesicles was $\sim 3 \mathrm{mg} / \mathrm{mL}$.

\section{Determination of size and zeta potential}

The average particle size and zeta potential of nanovesicles were determined by using a particle size analyzer (Malvern Zetasizer Nano ZS at an angle of $173^{\circ}$ ) at $25^{\circ} \mathrm{C} \pm 0.5^{\circ} \mathrm{C}$. The experiment was independently performed with three replicates per experimental group $(n=3)$. 


\section{Encapsulation efficiency}

The concentration of insulin was determined using reverse phase-HPLC (RP-HPLC) with an Agilent Technologies 1200 series HPLC system (Agilent, Santa Clara, CA, USA) and a 300 SB-C18 column $(4.6 \times 250 \mathrm{~mm}, 5 \mu \mathrm{m}$; Agilent). The measurement conditions were as follows: $0.2 \mathrm{M}$ sulfate buffer/acetonitrile (74:26, v/v), flow rate: $1 \mathrm{~mL} / \mathrm{min}$, ultraviolet detection: $214 \mathrm{~nm}$, injection volume: $20 \mu \mathrm{L}$, and column oven temperature: $40^{\circ} \mathrm{C}$.

The encapsulation efficiency (EE, \%) of the three nanovesicles was determined using a fast ultrafiltration method. Briefly, $1 \mathrm{~mL}$ of prepared nanovesicle suspension was placed in a centrifugal filter tube (Amicon Ultra-4 centrifugal devices, 100K nominal molecular weight limit; Millipore, Billerica, MA, USA) and centrifuged at 4,000 rpm for 40 minutes to separate the free and entrapped insulin. After ultrafiltration, the amount of insulin in the ultrafiltrates was measured using RP-HPLC. The centrifugal filter tube was washed three times with PBS at 2,500 rpm for 10 minutes, and the ultrafiltrate was collected and measured using RP-HPLC to determine the absorbed drug on the centrifugal filter tube. The EE was calculated according to the following equation:

$$
\mathrm{EE} \%=\left(\frac{W_{\text {total-insulin }}-\left(W_{\text {free-insulin }}+W_{\text {absorbed-insulin }}\right)}{W_{\text {total-insulin }}}\right) \times 100 \%
$$

where $W_{\text {total-insulin }}$ is the total amount of insulin in nanovesicles, $W_{\text {free-insulin }}$ is the amount of insulin in the ultrafiltrates, and $W_{\text {absorbed-insulin }}$ is the absorbed insulin on the centrifugal filter. The experiment was independently performed with three replicates per experimental group $(\mathrm{n}=3)$.

\section{Deformability of nanovesicles}

The deformability of nanovesicles was determined by a stainless steel pressure filter device. Three nanovesicles were extruded through $50 \mathrm{~nm}$ polycarbonate membranes at a constant pressure of $0.45 \mathrm{MPa}$ for 5 minutes. After extrusion, the average particle size of nanovesicles was measured using a particle size analyzer. The deformability index (DI) of nanovesicles was calculated using the following equation: ${ }^{20}$

$$
\mathrm{DI}=J *\left(\frac{r_{\mathrm{v}}}{r_{\mathrm{p}}}\right)^{2}
$$

where $J$ is the rate of penetration through a permeability membrane, $r_{\mathrm{v}}$ is the particle size after extrusion, and $r_{\mathrm{p}}$ is the pore diameter of the permeability membrane $(50 \mathrm{~nm})$.
The experiment was independently performed with three replicates per experimental group $(n=3)$.

\section{Structural analysis of nanovesicles}

\section{Transmission electron microscopy}

The morphology of nanovesicles was studied using TEM1400 plus at $120 \mathrm{kV}$. The nanovesicles were diluted 50 times and deposited on a carbon support film (Zhongjingkeyi Technology, Shanghai, People's Republic of China), allowed to stand for 5 minutes, and then the excess fluid was absorbed by a filter paper. The sample was then negatively stained by adding a drop of $1 \%$ phosphotungstic acid, allowed to stand for 5 minutes, and dried at $25^{\circ} \mathrm{C}$.

\section{Conformational stability}

To verify the integrity of the secondary structure of insulin entrapped in nanovesicles and the released insulin, circular dichroism (CD) studies were carried out. The released insulin of the three nanovesicles was obtained by using a fast ultrafiltration method. Briefly, after treatment with a moderate amount of ethanol, $1 \mathrm{~mL}$ of prepared nanovesicle suspension was placed in a centrifugal filter tube and centrifuged at $4,000 \mathrm{rpm}$ for 40 minutes to obtain the released insulin. The $\mathrm{CD}$ spectra were acquired at $25^{\circ} \mathrm{C}$ using a spectropolarimeter (J-815 Spectropolarimeter; Jasco, Osaka, Japan) in the far ultraviolet region in a $1 \mathrm{~mm}$ path length cell and by using a step size of $1 \mathrm{~nm}$ and a scan rate of $50 \mathrm{~nm} / \mathrm{min}$. The concentration of insulin was $15 \mu \mathrm{g} / \mathrm{mL}$. The lamp housing was purged with nitrogen, and an average of three scans was obtained; a reference scan of the relevant buffer was subtracted.

\section{In vitro mucosal permeation of nanovesicles \\ Preparation of porcine buccal mucosa}

Fresh pig tongue samples were collected from a slaughterhouse immediately after euthanasia, stored at $4^{\circ} \mathrm{C}$, and then transported to the laboratory. The porcine buccal mucosa was obtained using a drum dermatome (Lite Manufacturing Medical Devices Co., Ltd, Shanghai, People's Republic of China). The thickness of the mucosa used as a barrier membrane in this study was $\sim 0.7 \mathrm{~mm}$. The prepared porcine buccal mucosa was then stored in a lyophilized protective solution ${ }^{21}$ ( $4 \%$ albumin, $10 \%$ dimethyl sulfoxide, and $86 \% \mathrm{PBS}$ ) in a refrigerator at $-20^{\circ} \mathrm{C}$ until use.

\section{Mucosal permeation study}

Mucosal permeation studies were performed using a Franz diffusion chamber (PermeGear, Hellertown, PA, USA) consisting of horizontal diffusion chambers, magnetic 
stirring control system, and heating circulation system with a programmable temperature control device. The temperature of the water bath was maintained at $37^{\circ} \mathrm{C} \pm 0.5^{\circ} \mathrm{C}$, the effective permeation area was $1.77 \mathrm{~cm}^{2}$, and the receptor and donor chamber volumes were both $3.4 \mathrm{~mL}$. The buccal mucosa sample was mounted between the donor and receptor chambers, with the epithelial side facing the donor chamber. PBS (pH 7.4) was used as the receiver medium, and undiluted nanovesicles were used as the donor solution. The receiver medium was stirred using a magnetic bar at a rate of $500 \mathrm{rpm}$, and $100 \mu \mathrm{L}$ of the sample was withdrawn through the sampling port at predetermined times $(0,0.5,1,1.5,2,3$, and 4 hours). The receiver medium was immediately replenished with an equal volume of PBS. The collected samples were analyzed using HPLC for insulin content. Flux and $\mathrm{P}_{\text {app }}$ were calculated using the following mathematical expressions:

$$
P_{\text {app }}=\frac{(\mathrm{dQ} / \mathrm{dt})}{\mathrm{A} \times \Delta \mathrm{C}}
$$

$\mathrm{dQ} / \mathrm{dt}$ is the cumulative amount of insulin permeated the mucosa per unit time, $\Delta \mathrm{C}$ is the concentration difference of insulin between the donor and receiver chambers (approximately equal to the initial drug donor concentration), and $\mathrm{A}$ is the surface area of diffusion $\left(\mathrm{cm}^{2}\right)$. The experiment was independently performed with three replicates per experimental group $(n=3)$.

After mucosal permeation study, the concentration of insulin retained in the mucosa was measured. The mucosal samples were collected from the diffusion chambers, washed with PBS, and dried with filter paper. To measure the effective permeation area, the samples were cut into small pieces, soaked in $1 \mathrm{~mL}$ PBS solution for 12 hours, sonicated for 5 minutes, and the clear supernatant was analyzed using HPLC. The experiment was independently performed with three replicates per experimental group $(n=3)$.

\section{Receiver medium visualization using TEM}

TEM was used to investigate the receiver medium after permeation for 4 hours to determine the intact or disintegrated nanovesicles. The receiver medium was filtered through a nylon membrane $(0.45 \mu \mathrm{m}$ pore size $)$ to remove contaminants, and the preparation process was the same as that used for the previous TEM.

\section{CLSM studies}

CLSM was performed to evaluate the depth and path of the nanovesicle transport. Three nanovesicles were labeled with two types of fluorescence markers, rhodamine and FITC
(RF-IPC-DNVs, RF-INS-DNVs, and RF-IPC-NVs). They were applied to the in vitro mucosa permeation model for 2 and 4 hours. After the experiment, the mucosa was washed with PBS. Excess formulation was wiped off with a filter paper, and the mucosa was then frozen with liquid nitrogen and stored at $-80^{\circ} \mathrm{C}$ before slicing. The frozen mucosa was vertically sectioned into $5 \mu \mathrm{m}$ slices by a cryostat microtome. The distribution of fluorescence in the mucosa was observed by CLSM. FITC-labeled insulin (green fluorescence) was measured at excitation and emission wavelengths of 490 and $525 \mathrm{~nm}$, respectively. Rhodamine-labeled phospholipid (red fluorescence) was measured at excitation and emission wavelengths of 557 and $581 \mathrm{~nm}$, respectively.

\section{Influence of IPC and free phospholipid on insulin absorption}

To investigate the influence of IPC and free phospholipid on the absorption of insulin further, IPC $(3 \mathrm{mg} / \mathrm{mL})$ and a physical mixture of insulin and phospholipid (Phy-IP) were prepared. For Phy-IP, a weighed amount of insulin and phospholipid (1:20, w/w) were dissolved in PBS to obtain an insulin concentration of $3 \mathrm{mg} / \mathrm{mL}$. Then, the mucosal permeation study was performed as described in the "Mucosal permeation study" section.

\section{In vivo bioactivity and hypoglycemic effect of nanovesicles}

The relative pharmacological bioactivity of insulin delivered by buccal administration of IPC-DNVs, INS-DNVs, and IPC-NVs was evaluated in normal rabbits by assessing the hypoglycemic effect. To prevent animals from swallowing the dosing solution, the esophagus of each rabbit was surgically ligated. The esophagus was untied 30 minutes after administration of the drug solution.

Before the experiment, the rabbits were fasted for 2 hours, but allowed free access to water. The rabbits were randomly divided into groups of three each: the control received normal saline via the mucosa; the insulin-Sub group received insulin solution by subcutaneous injection (1 IU/kg); the IPC-DNVSub group received IPC-DNVs by subcutaneous injection (1 IU/kg); and IPC-DNVs, INS-DNVs, and IPC-NVs were administered through the mucosa (10 IU/kg).

Blood glucose levels of samples collected from the rabbit ear veins at 30-minute intervals from 0 to 6 hours after administration were measured using a blood analyzer (OneTouch Ultra; Johnson \& Johnson, New Brunswick, NJ, USA). Furthermore, the relative pharmacological bioavailability (Fp) of insulin after buccal administration was calculated according to the following equation: 


$$
\mathrm{F}_{\mathrm{p}}=\frac{\mathrm{AAC}_{\text {buccal }} \times \text { Dose }_{\text {s.c. }}}{\mathrm{AAC}_{\text {s.c. }} \times \text { Dose }_{\text {buccal }}} \times 100 \%
$$

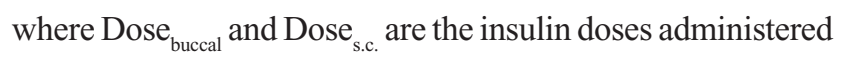
buccally and subcutaneously, respectively, and AAC values are the area above the curve values of reduction in blood glucose level over time.

\section{In vivo safety studies}

Acute irritation caused by buccal administration of IPCDNVs was determined by histopathologic studies. The rabbits were randomly divided into two groups: group 1, buccal administration of IPC-DNVs (10 IU $/ \mathrm{kg}$ ) and group 2, buccal administration of normal saline at a volume equal to that of group 1. To prevent the animals from swallowing the dosing solution, the esophagus of each rabbit was surgically ligated. IPC-DNVs and normal saline were administered three times at 4-hour intervals (for repeat test). After 12 hours, the rabbits were sacrificed and the tongues were removed. Hematoxylin and eosin staining was performed to detect histopathologic changes. The experiment was independently performed with three replicates per experimental group $(n=3)$.

\section{Statistical analysis}

Data are expressed as the mean $\pm \mathrm{SD}$ of three independent experiments. Student's $t$-test was applied using SPSS software (SPSS Inc., Chicago, IL, USA) to determine the statistically significant differences between groups. A $P$-value $<0.05$ was considered statistically significant $(* P<0.05, * * P<0.01$, and $* * * P<0.001)$.

\section{Results}

\section{Physicochemical characterization of nanovesicles}

The particle size, zeta potential, EE, and DI values of IPCDNVs, INS-DNVs, and IPC-NVs are shown in Table 1. All three kinds of nanovesicles showed good dispersion (Figure 2). During the deformation process, the $r_{\mathrm{v}}$ of IPC-NVs was smaller than that before the process, whereas those of
IPC-DNVs and INS-DNVs were almost the same, indicating that IPC-NVs were hard to deform to penetrate the mucosa.

\section{Structural analysis of nanovesicles}

\section{Transmission electron microscopy}

IPC-DNVs showed a well-defined inner aqueous phase surrounded by a lipid bilayer with an inhomogeneous thickness (Figure 2). In contrast, INS-DNVs had a thinner shell than that of IPC-DNVs and IPC-NVs. It can be clearly seen that IPC-DNVs had obvious whorls, whereas INSDNVs did not have. However, IPC-NVs were significantly different from IPC-DNVs and INS-DNVs, and their lipid bilayer was circular and had a well-defined structure. Furthermore, the particle size of IPC-NVs was slightly larger than that of nanovesicles that were spherical or ellipsoid.

\section{Conformational stability}

Figure 3 shows the CD spectra of free insulin solution, insulin entrapped into the nanovesicles, and the released insulin. Their CD spectra showed two minima at about 208 and $222 \mathrm{~nm}$, which were typical of $\alpha$-helix structure. This was in close agreement with the spectra obtained by others. ${ }^{22,23}$ All of the released insulin had a CD spectrum very similar to the CD spectrum of free insulin. Furthermore, the $\alpha$-helix ratio was between $23.3 \%$ and $28.4 \%$, which was in agreement with that of free insulin $(23.5 \%)$. While the spectra observed from insulin encapsulated into nanovesicles had some changes, the $\alpha$-helix ratio of the three nanovesicles was much higher than that of the free insulin solution (23.5\%). The $\alpha$-helix ratios for IPC-DNVs, INS-DNVs, and IPC-NVs were $46.6 \%, 67.5 \%$, and $51.9 \%$, respectively.

\section{In vitro mucosal permeation of nanovesicles \\ Mucosal permeation study}

Figure 4A and B shows the flux and insulin deposited in the mucosa after the permeation study, respectively. The flux of nanovesicles was ranked as follows: IPC-DNVs $>$ INSDNVs $>$ IPC-NVs. The $\mathrm{P}_{\text {app }}$ of insulin was determined to be $(2.71 \pm 0.14) \times 10^{-6},(2.15 \pm 0.25) \times 10^{-6}$, and $(1.58 \pm 0.13) \times 10^{-6} \mathrm{~cm} / \mathrm{s}$, respectively, in the steady state. IPC-DNVs induced a much higher insulin deposition than INS-DNVs and IPC-NVs.

Table I Physicochemical characteristics of IPC-DNVs, INS-DNVs, and IPC-NVs

\begin{tabular}{l|l|l|l|l|l}
\hline Formulation & Size $(\mathbf{n m})$ & $\begin{array}{l}\text { Zeta potential } \\
(\mathbf{m V})\end{array}$ & EE & $\boldsymbol{r}_{\mathrm{v}}(\mathbf{n m})$ & $\mathbf{D I}\left(\mu \mathrm{g} / \mathbf{c m}^{2} / \mathbf{s}\right)$ \\
\hline IPC-DNVs & $85.84 \pm 2.38$ & $-26.2 \pm 0.5$ & $77.47 \% \pm 3.85 \%$ & $86.20 \pm 1.35$ & $38.72 \pm 2.35$ \\
INS-DNVs & $89.05 \pm 2.81$ & $-32.0 \pm 0.8$ & $72.49 \% \pm 2.48 \%$ & $89.49 \pm 1.95$ & $42.97 \pm 1.95$ \\
IPC-NVs & $94.67 \pm 2.40$ & $-45.3 \pm 0.7$ & $79.27 \% \pm 4.24 \%$ & $80.27 \pm 3.16$ & $2.64 \pm 0.17 * * *$ \\
\hline
\end{tabular}

Notes: Data are presented as the mean \pm SD $(n=3)$. ***P $<0.00$ I between IPC-DNVs and IPC-NVs.

Abbreviations: DI, deformability index; DNVs, deformable nanovesicles; EE, encapsulation efficiency; INS, insulin; IPC, insulin-phospholipid complex; NVs, conventional nanovesicles; $r_{\mathrm{v}}$, the particle size after extrusion. 

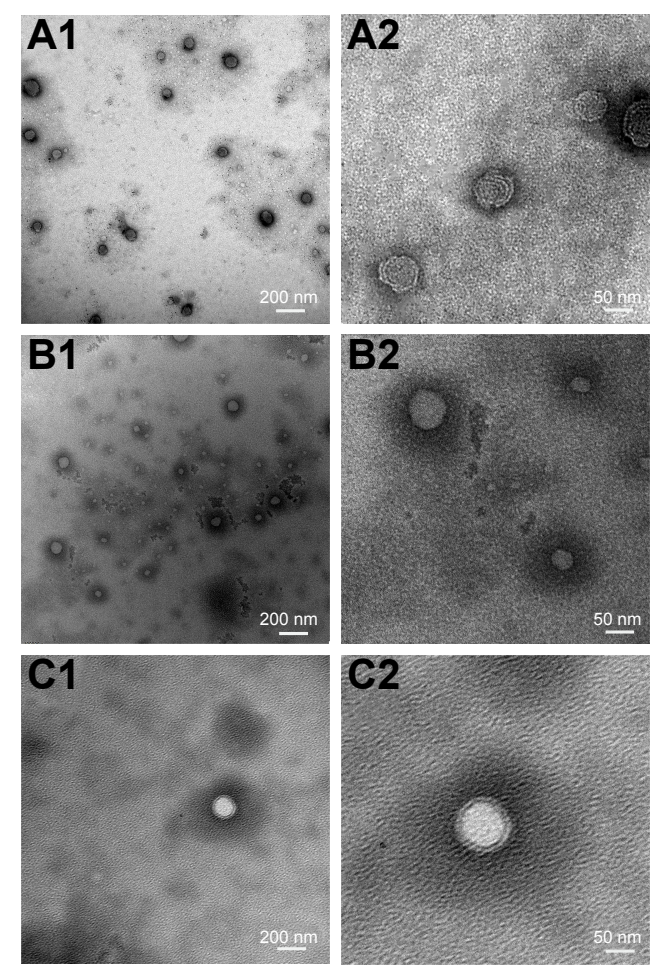

\section{A3}

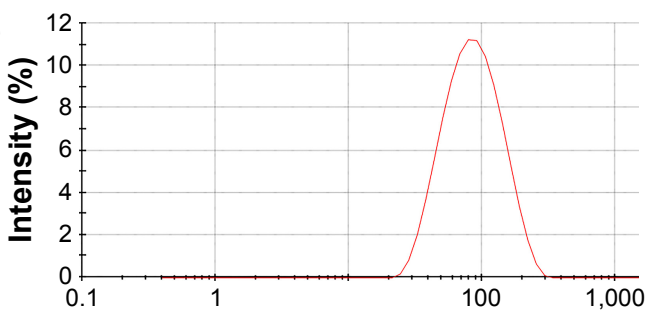

B3

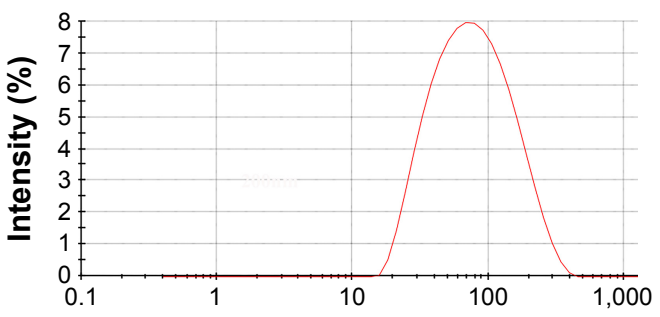

C3

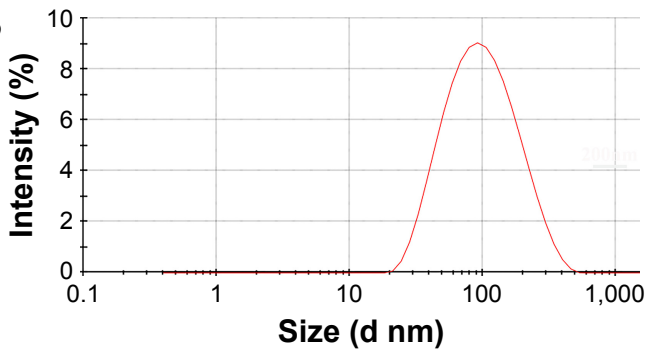

Figure 2 Morphology and size distribution of (A) IPC-DNVs, (B) INS-DNVs, and (C) IPC-NVs.

Notes: I refers to morphology at low magnification; 2 refers to morphology at high magnification; 3 refers to size distribution.

Abbreviations: DNVs, deformable nanovesicles; INS, insulin; IPC, insulin-phospholipid complex; NVs, conventional nanovesicles.

\section{Receiver medium visualization}

The TEM images of IPC-DNVs, INS-DNVs, and IPC-NVs are shown in Figure 5. The receiver medium of IPC-NVs showed no detectable levels, whereas IPC-DNVs and INSDNVs were spherical or ellipsoid, indicating that the DNVs (IPC-DNVs and INS-DNVs) penetrated the mucosa to reach

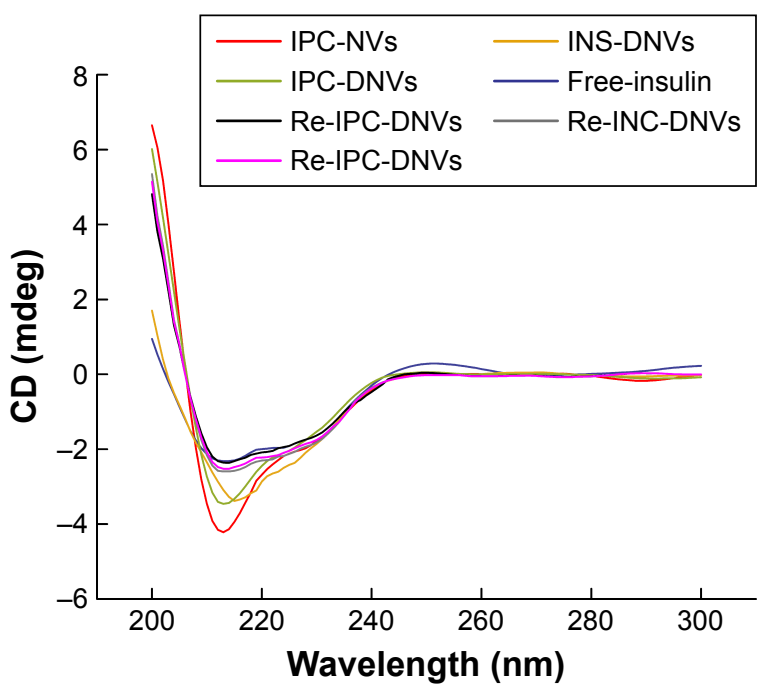

Figure $3 \mathrm{CD}$ spectra of insulin entrapped in nanovesicles and the released insulin. Abbreviations: CD, circular dichroism; DNVs, deformable nanovesicles; INS, insulin; IPC, insulin-phospholipid complex; NVs, conventional nanovesicles. the receiver medium as intact nanovesicles. Moreover, the IPC-DNV shells were thinner than they were before penetration, and they seemed to be stained in the core of the nanovesicles, whereas INS-DNVs were not different from their previous state.

\section{CLSM study}

Figure 6 illustrates the fluorescence intensity of insulin (green) and phospholipids (red) at 2 and 4 hours after treatment. IPC-NVs did not change much with time and only remained in the upper layer of the epidermis. For INS-DNVs, the change in red fluorescence was higher than that in green fluorescence, whereas for IPC-DNVs, the change between red and green fluorescence was consistent. Figure 6B-e and $-\mathrm{f}$ shows that IPC-DNVs and INS-DNVs penetrated the mucosa of full thickness since the fluorescence was detected throughout the tissue. In contrast, the fluorescence intensity of IPC-DNVs was higher than that of INS-DNVs at every depth of the mucosa. Moreover, both intracellular and extracellular fluorescence intensities of IPC-DNVs were almost the same (Figure 6C). The IPC-DNVs showed higher fluorescence intensity than INS-DNVs and IPC-NVs, which was in accordance with the results of the in vitro mucosal deposition studies. 

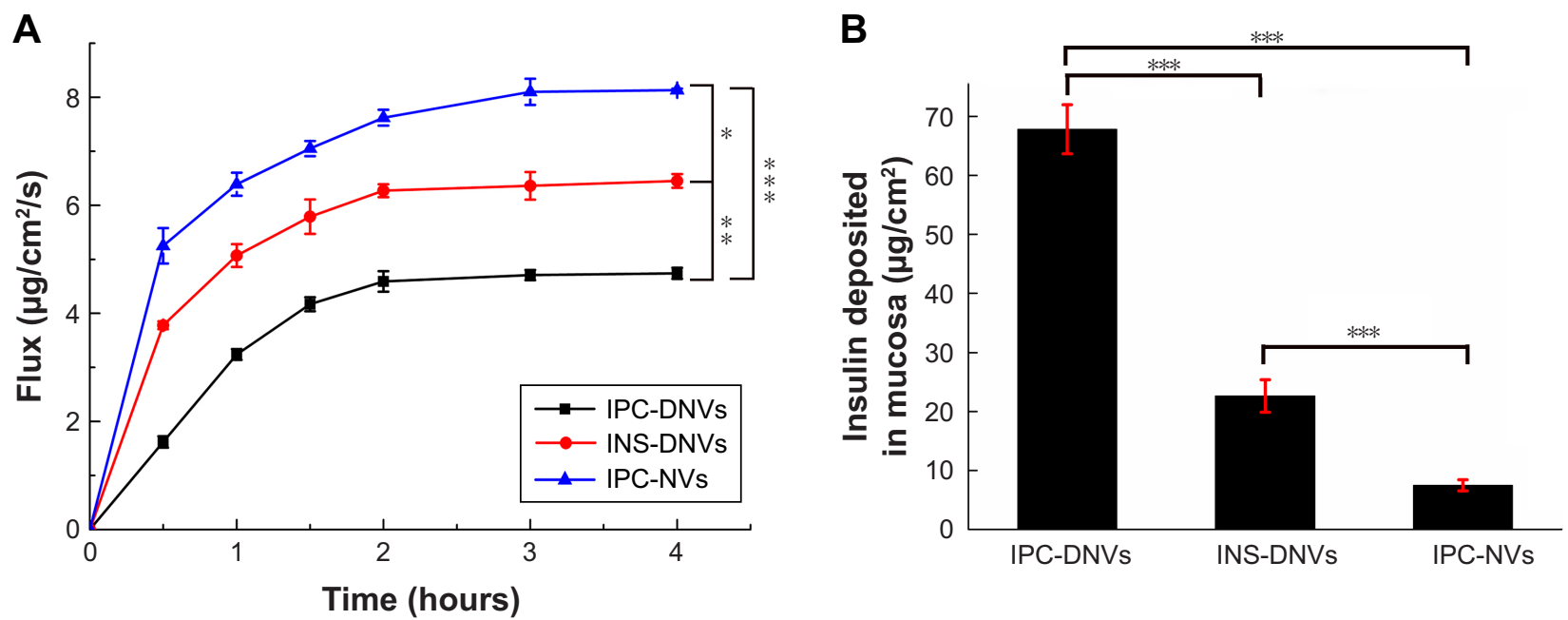

Figure 4 Mucosal permeation profiles of IPC-DNVs, INS-DNVs, and IPC-NVs.

Notes: (A) The mucosa permeation profile; (B) insulin deposited in full-thickness mucosa $\left(* P<0.05, * * P<0.01,{ }^{*} * * P<0.00 I ; n=3\right)$.

Abbreviations: DNVs, deformable nanovesicles; INS, insulin; IPC, insulin-phospholipid complex; NVs, conventional nanovesicles.

\section{Influence of IPC and free phospholipid on insulin absorption}

As presented in Table 2, the flux and $\mathrm{P}_{\text {app }}$ of the Phy-IP showed no significant difference with insulin and were significantly lower than those of IPC. This demonstrated that free phospholipid could barely increase the buccal permeability of insulin. The hydrogen bonds between insulin and the phospholipid, which led to a small particle size and good solubility and permeability of IPC, might be the reason for the improved absorption.

\section{In vivo bioactivity and hypoglycemic effect of nanovesicles}

The hypoglycemic effects of normal saline (control), IPCDNVs, INS-DNVs, and IPC-NVs after buccal administration and subcutaneous administration are shown in Figure 7. Slight changes in the secondary structure of insulin have little effect on the biological activity of insulin. A significant difference in blood glucose reduction was observed between rabbits administered IPC-DNVs and INS-DNVs, confirming their hypoglycemic effect. After the subcutaneous injection of insulin, the blood glucose level was reduced by $\sim 53 \%$ of the initial level after 1 hour. The maximal hypoglycemic effects of IPC-DNVs (70\%) and INS-DNVs $(30 \%)$ were observed at 2.5 and 1 hour, respectively. IPC$\mathrm{NVs}$ were not different from the control and showed no hypoglycemic effect. Moreover, buccal administration of IPC-DNVs demonstrated prolonged glucose reduction and the hypoglycemic effect lasted for 4 hours. The Fp of insulin significantly increased through IPC-DNVs compared to
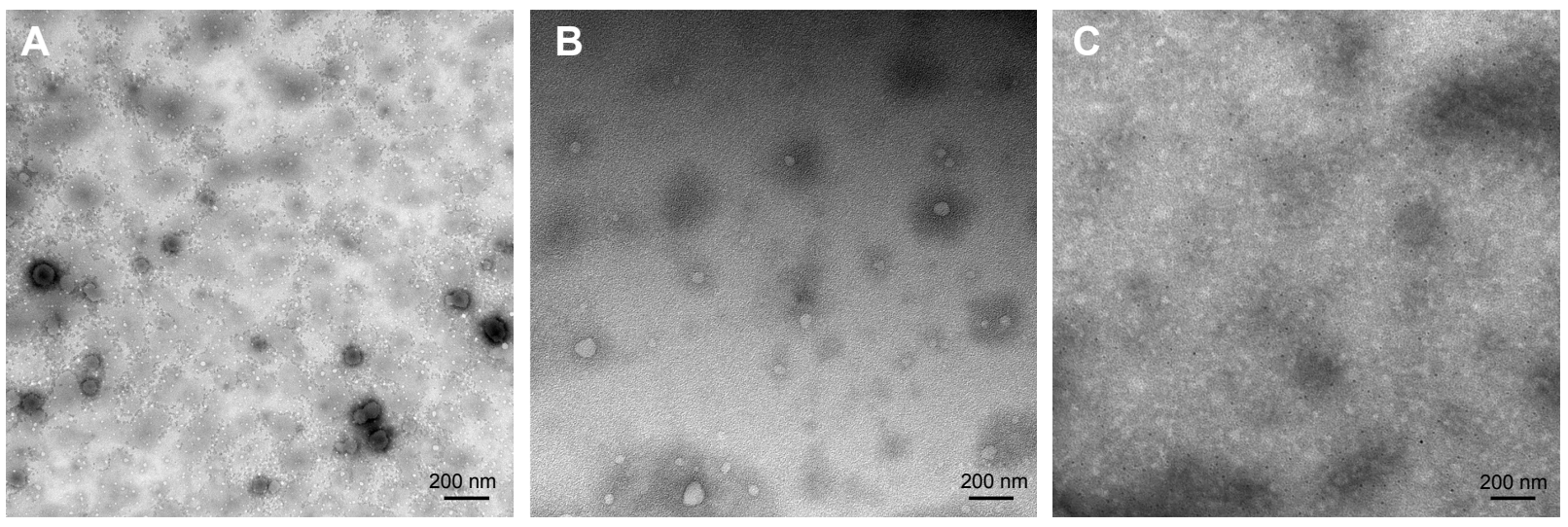

Figure 5 Transmission electron microscopy images of the receiver medium after mucosal penetration study of (A) IPC-DNVs, (B) INS-DNVs, and (C) IPC-DNVs at 4 hours.

Abbreviations: DNVs, deformable nanovesicles; INS, insulin; IPC, insulin-phospholipid complex; NVs, conventional nanovesicles. 

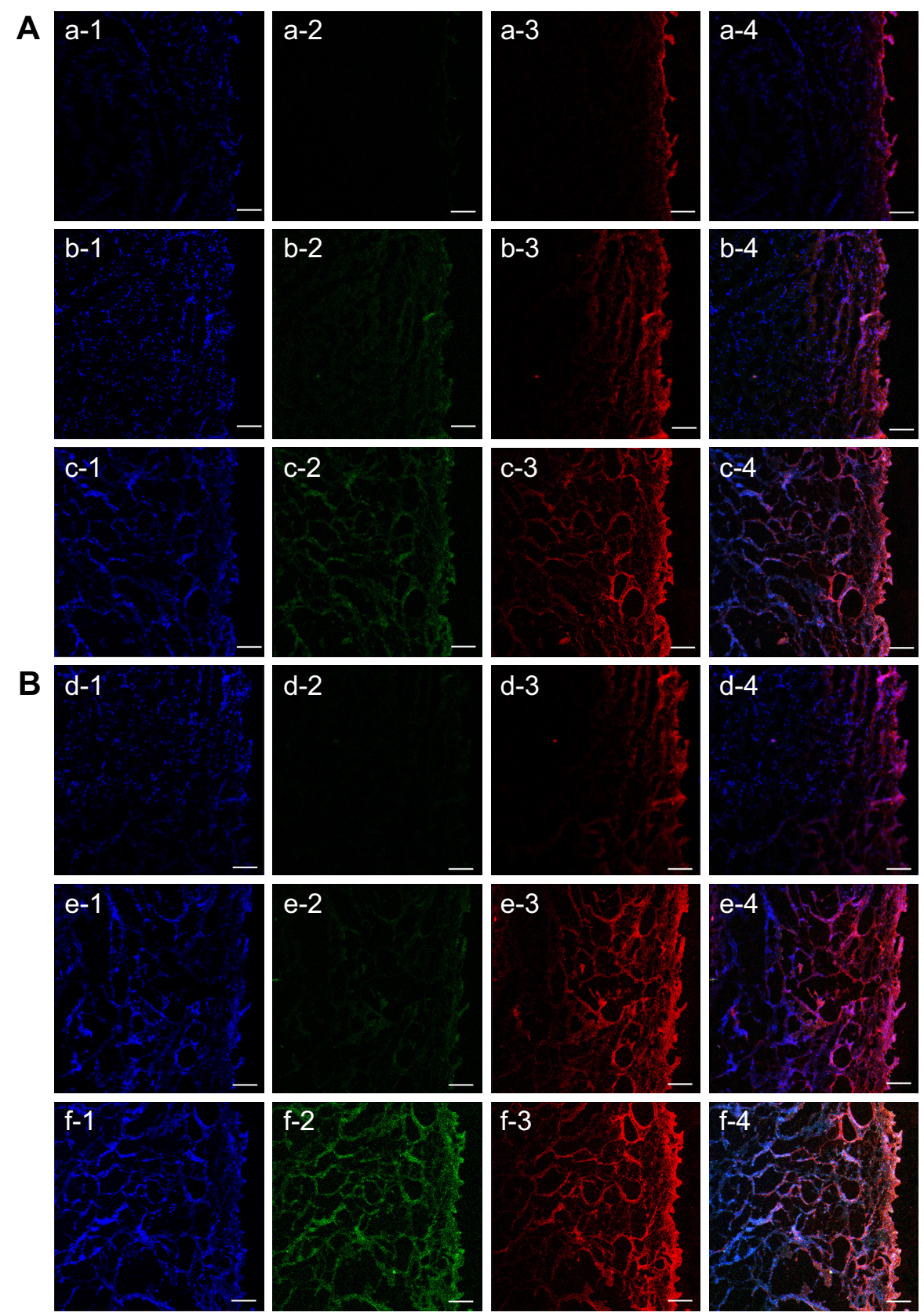

C

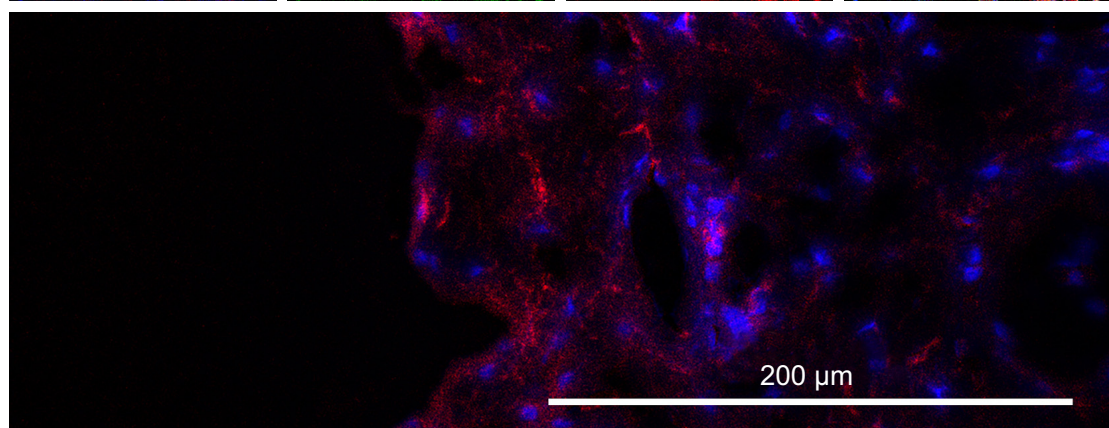

Figure 6 Confocal laser scanning microscopy of mucosa after treatment for 2 and 4 hours.

Notes: (A) After 2-hour treatment with (a) RF-IPC-NVs, (b) RF-INS-DNVs, and (c) RF-IPC-DNVs. (B) After 4-hour treatment with (d) RF-IPC-NVs, (e) RF-INS-DNVs, and (f) RF-IPC-DNVs. (C) Magnified mucosa after 2-hour treatment with R-IPC-DNVs. -I refers to the channels of DAPI; -2 refers to the channels of FITC; -3 refers to the channels of rhodamine I; -4 refers to the merged image. The bar of $A$ and $B$ is $100 \mu \mathrm{m}$.

Abbreviations: DAPI, 4',6-diamidino-2-phenylindole; DNVs, deformable nanovesicles; FITC, fluorescein isothiocyanate; INS, insulin; IPC, insulin-phospholipid complex; $\mathrm{NVs}$, conventional nanovesicles; RF, rhodamine-labeled phospholipid and FITC-labeled insulin. 
Table 2 Influence of IPC and free phospholipid on insulin absorption

\begin{tabular}{l|l|l|l}
\hline & IPC & Phy-IP & Insulin \\
\hline Flux $\times 10^{-3} \mu \mathrm{g} / \mathrm{cm}^{2} / \mathrm{s}$ & $5.77 \pm 0.38^{*}$ & $3.19 \pm 0.22$ & $3.07 \pm 0.44$ \\
$\mathrm{P}_{\text {app }} \times 10^{-6} \mathrm{~cm} / \mathrm{s}$ & $1.99 \pm 0.42^{*}$ & $1.10 \pm 0.26$ & $1.06 \pm 0.48$ \\
\hline
\end{tabular}

Notes: Data are presented as the mean \pm SD $(n=3)$. $* P<0.05$ between IPC and insulin.

Abbreviations: flux, the absorption rate constant; IPC, insulin-phospholipid complex; $\mathrm{P}_{\text {app }}$, apparent permeability coefficient; Phy-IP, physical mixture of insulin and phospholipid.

INS-DNVs and IPC-NVs (15.53\% vs $3.09 \%$ and $1.96 \%$, respectively).

\section{In vivo safety studies}

At 12 hours after the administration (three times), there were no systemic adverse reactions in IPC-DNVs and normal saline. The results of slice panoramic scanning are shown in Figure 8. The sublingual mucosa had no congestion, hemorrhage, swelling, erosion, or ulcer. There were no abnormalities in the basal layer, lamina propria, submucosal tissue, or muscle fibers. Therefore, no visible mucosal irritation occurred after repeated buccal administration of IPC-DNVs when compared with the control.

\section{Discussion}

In this study, we evaluated the particle size, zeta potential, DI, and morphology of IPC-DNVs, INS-DNVs, and IPC-NVs. We found that their initial particle size was in the range of $80-90 \mathrm{~nm}$. However, compared with INS-DNVs, IPC-DNVs

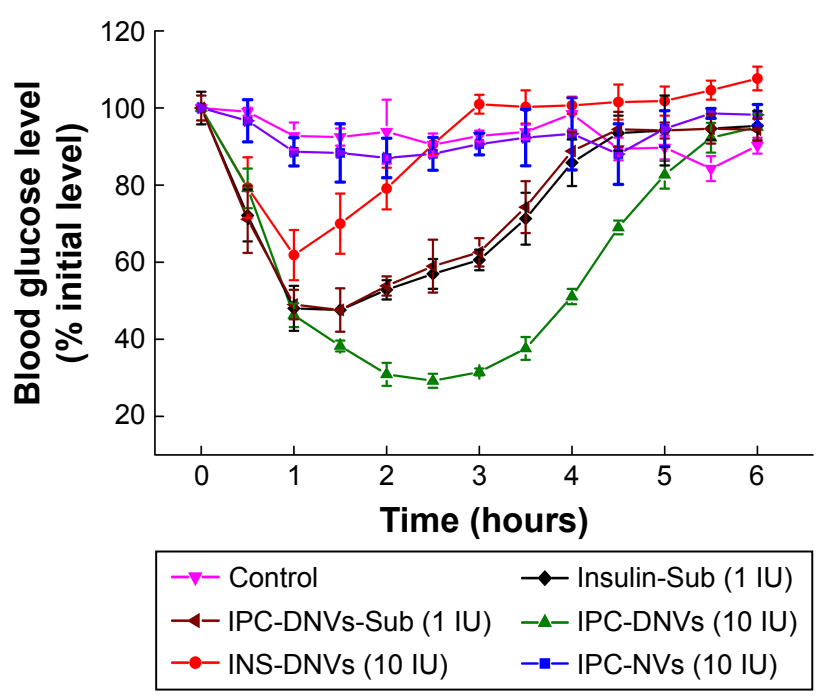

Figure $\mathbf{7}$ In vivo hypoglycemic effect and bioactivity of IPC-DNVs, INS-DNVs, and IPC-NVs $(n=3)$.

Note: Data are presented as the mean $\pm S D(n=3)$.

Abbreviations: DNVs, deformable nanovesicles; INS, insulin; IPC, insulinphospholipid complex; NVs, conventional nanovesicles; Sub, subcutaneous. had a thicker shell, which indicated the IPC might mainly be present on both sides of the shell rather than in the interior water chamber of the nanovesicles. ${ }^{24}$ Furthermore, the IPC of IPC-DNVs may not be homogeneously distributed because the thickness of the lipid bilayer was not uniform. Compared with IPC-DNVs, IPC-NVs had a higher zeta potential and lower DI value. The higher zeta potential indicated higher stability of nanovesicles, which was consistent with its strong spherical structure. The high DI values of IPC-DNVs and INS-DNVs were mainly caused by edge activators. Edge activators could disrupt the phospholipid bilayers when they are inserted into the bilayers. In this study, SDC and Tween 20 were used in combination as edge activators to form nanovesicles with high deformability. ${ }^{25}$

From the CD spectra, peaks observed from the released and free insulin solutions were observed at similar wavelengths, indicating the well-preserved conformational stability of insulin after entrapment into the various nanovesicles and release from nanovesicles. It might be the main reason why insulin maintained its bioactivity. When insulin was encapsulated into the nanovesicles, the $\mathrm{CD}$ spectra showed some difference with free insulin and the $\alpha$-helix ratio increased. The increased $\alpha$-helix ratio might mainly be caused by the compression of insulin structure. For IPCDNVs and IPC-NVs, the result of the secondary structure indicated an increase in $\alpha$-helix ratio, reaching $46.6 \%$ and $51.9 \%$, respectively. For IPC-DNVs, this might have resulted from the spatial volume of IPC. When IPC became an integral part of the membrane during formation, ${ }^{10,26}$ the structure of insulin became tighter, resulting in an increased of $\alpha$-helix ratio. For INS-DNVs, insulin was encapsulated in the interior water chamber, which might have been due to the formation of micelle-like structures or their aggregates by insulin, ${ }^{27}$ so the structure of insulin became much tighter, leading to increased $\alpha$-helix ratio. Although there were some changes in the secondary structure of insulin after forming nanovesicles, the system seemed to be stable to carry insulin and release it, simultaneously maintaining the activity of insulin (data can be seen in Figure 7, IPC-DNVs-Sub). Accordingly, we suggested the schematic of the three nanovesicles in Figure 1.

In the in vitro study, the mucosa was treated with IPCDNVs, INS-DNVs, and IPC-NVs for 4 hours as all formulations had reached a steady state at that point. A comparison of IPC-DNVs and IPC-NVs revealed that the flux, $\mathrm{P}_{\text {app }}$, and deposition of insulin in IPC-DNVs were much higher than those in IPC-NVs, when they both contained the IPC. This difference might have been caused by the deformable structure of IPC-DNVs, which enabled them to penetrate 

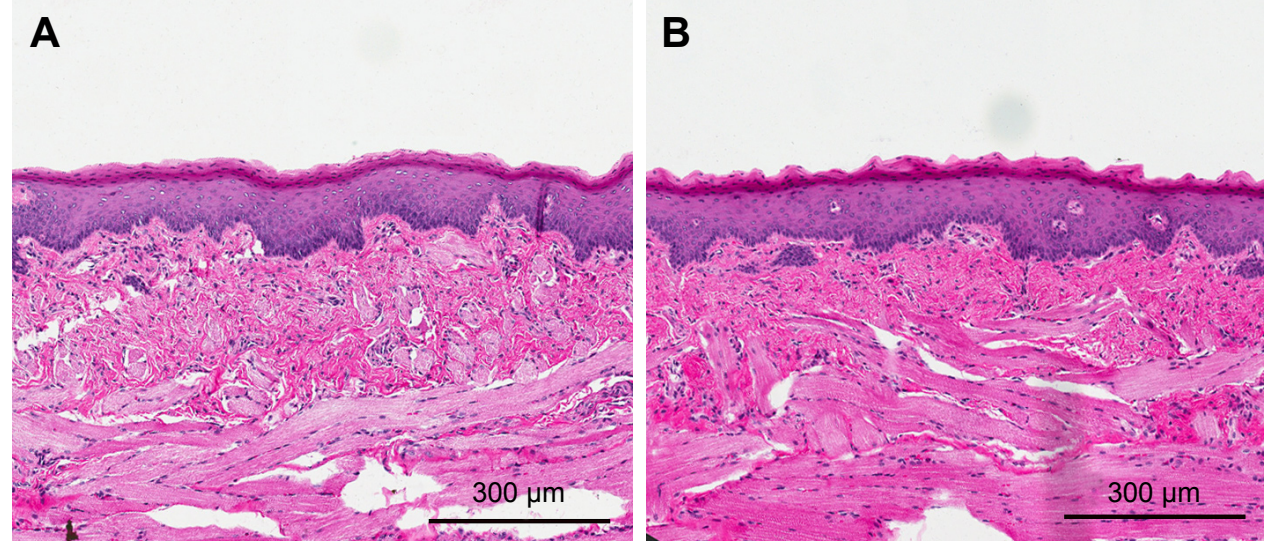

Figure 8 Optical microscopy images of hematoxylin and eosin stained sections of sublingual mucosa for administration of (A) normal saline and (B) IPC-DNVs. Abbreviations: DNVs, deformable nanovesicles; IPC, insulin-phospholipid complex.

the permeability barrier of the epithelium. A comparison of IPC-DNVs and INS-DNVs showed that they had similar deformability, whereas the flux, $\mathrm{P}_{\text {app }}$, and deposition of IPCDNVs were higher than those of INS-DNVs. These might be attributed to two factors. First, we proposed that the IPC encapsulated in the shell of IPC-DNVs was easily dropped during the penetration process, and then it was fused with the cell membrane, which increased the deposition of IPC. The deposited IPC involved the sequential penetration of cells until it entered the systemic circulation to increase the flux and $\mathrm{P}_{\text {app }}$. Another factor was free IPC (according to the EE of $23 \%$ ), which played a significant role compared with insulin and Phy-IP. Therefore, we inferred that IPC could have a penetration-enhancing effect.

TEM could detect intact nanovesicles in the receptor chamber, which indicated that the nanovesicles might be transferred through the mucosa in an intact form. INS-DNVs were not significantly different after penetrating the mucosa. The shell of IPC-DNVs became thinner because IPC may have been dropped during membrane penetration. Staining was evident in the core of nanovesicles, possibly due to the migration of insulin during transmembrane penetration. A vesicle must lose some of its content during deformation in a pore because high deformability increases the leakage rate..$^{28}$ This compensated for the volume difference between a sphere and an ellipsoid, or any other non-spherical shape adapted to the pore size. ${ }^{29}$ In practice, such a volume exchange would cause the dropped IPC to be absorbed or fused with the mucosa. These observations indicated that IPC-DNVs could act as a drug carrier that can be transported to the mucosa in an intact form.

CLSM results indicated that undeformable nanovesicles, such as IPC-NVs, could not penetrate the deep layer of the epidermis and only remained at the upper layer of the epidermis. In contrast, the DNVs, namely IPC-DNVs and INS-DNVs, effectively permeated the viable epidermis as observed by the high fluorescence intensity in the mucosa. When 2-hour treatment was compared with 4-hour treatment, the synchronized change in red and green fluorescence showed that IPC-DNVs penetrated the mucosa in the form of IPC, while INS-DNVs showed a marked change in red fluorescence than in green fluorescence, indicating that the transport and deposition of free insulin in the mucosa were difficult. This observation was consistent with the deposition result, which might be caused by IPC deposited during the penetration process. It has been reported that the intercellular space of the mucosa (which widens close to the basement membrane) was the delivery pathway of large peptides such as insulin. ${ }^{30}$ Interestingly, the fluorescence was intense both intracellularly and intercellularly. Transcellular transport may have been mainly mediated by the IPC according to our research and paracellular transport may have been done by the DNVs. The possible mechanisms of mucosal penetration of IPC-DNVs are shown in Figure 9.

In our study, we discovered that IPC could maintain its biological activity in vivo. In vivo experiments with a rabbit esophagus ligation model demonstrated a significant improvement in the Fp of IPC-DNVs. The reason might lie in the different structures caused by IPC and deformability. The maximum concentration of IPC-DNVs showed a slight time lag compared with that of INS-DNVs, which might be caused by IPC deposited in the mucosa. This process involved the drug penetrating sequentially through the cells until it entered the systemic circulation, and this result is consistent with the in vitro mucosal permeation experiment. 


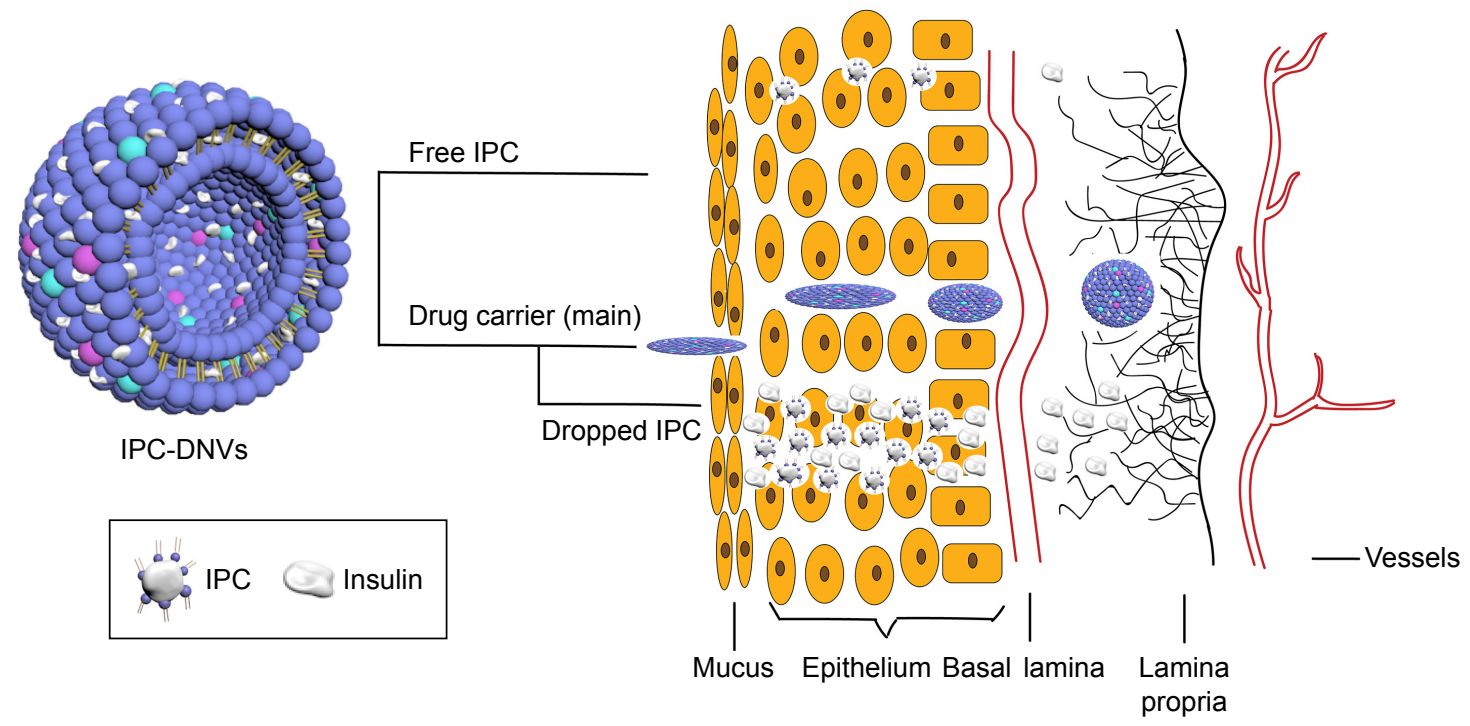

Figure 9 Mechanisms of IPC-DNV transport in mucosal permeation.

Abbreviations: DNVs, deformable nanovesicles; IPC, insulin-phospholipid complex.

The acute irritation studies indicated that buccal administration of IPC-DNVs resulted in no visible mucosal irritation to the buccal mucosa. Since the buccal mucosa is less sensitive to irritation and damage than other mucosa ${ }^{16,31}$ and may easily recover after slight irritation and damage, it is suitable for long-term administration. In addition, the edge activators Tween 20 and SDC were safe and have been proved to cause less irritation on the mucosa, ${ }^{32,33}$ and the other materials used in the preparation are nontoxic. In view of the no negative effects obtained in the study, the developed IPC-DNVs are considered safe for buccal use.

\section{Conclusion}

We developed a novel protein delivery strategy (IPC-DNVs) for enhancing the efficacy of buccal mucosal delivery. Compared with INS-DNVs and IPC-NVs, our IPC-DNV formulation significantly improved the buccal absorption of insulin. Notably, the Fp of IPC-DNVs reached $15.53 \%$, the maximum blood glucose level dropped to $30 \%$, and the hypoglycemic effect lasted for 4 hours. Therefore, the combined use of DNVs and IPC proves to be an efficient strategy to enhance the buccal bioavailability of difficult-to-deliver drugs. In vitro and in vivo investigations were conducted to elucidate the internal mechanisms. It was observed that IPC-DNVs did not cause negative effects on the mucosa. Based on our results, the enhanced buccal absorption of IPC-DNVs was attributed to the ability of DNVs to penetrate the main barrier, followed by the deposition of IPC, which enhanced buccal absorption. Our mechanisms could be a reference to understand other nanocarriers based on protein (peptide)-phospholipid complex that penetrate the mucosa and provide a theoretical basis for their development.

\section{Disclosure}

The authors report no conflicts of interest in this work.

\section{References}

1. Li J, Wang X, Zhang T, et al. A review on phospholipids and their main applications in drug delivery systems. Asian J Pharm Sci. 2015; 10(2):81-98.

2. Semalty A, Semalty M, Singh D, et al. Development and characterization of aspirin-phospholipid complex for improved drug delivery. Int $J$ Pharm Sci Nanotechnol. 2010;3(2):940-947.

3. Ruan J, Liu J, Zhu D, et al. Preparation and evaluation of selfnanoemulsified drug delivery systems (SNEDDSs) of matrine based on drug-phospholipid complex technique. Int J Pharm. 2010;386(1-2): 282-290.

4. Allen TM, Cullis PR. Liposomal drug delivery systems: from concept to clinical applications. Adv Drug Deliv Rev. 2013;65(1):36-48.

5. Hippalgaonkar K, Majumdar S, Kansara V. Injectable lipid emulsions advancements, opportunities and challenges. AAPS PharmSciTech. 2010;11(4):1526-1540.

6. Rupp C, Steckel H, Müller BW. Solubilization of poorly water-soluble drugs by mixed micelles based on hydrogenated phosphatidylcholine. Int J Pharm. 2010;395(1-2):272-280.

7. Bhattacharya S. Phytosomes: the new technology for enhancement of bioavailability of botanicals and nutraceuticals. Int $J$ Health Res. 2009;2(3):225-232.

8. Peng Q, Zhang ZR, Gong T, Chen GQ, Sun X. A rapid-acting, longacting insulin formulation based on a phospholipid complex loaded PHBHHx nanoparticles. Biomaterials. 2012;33(5):1583-1588.

9. Cui F, Shi K, Zhang L, Tao A, Kawashima Y. Biodegradable nanoparticles loaded with insulin-phospholipid complex for oral delivery: preparation, in vitro characterization and in vivo evaluation. $J$ Control Release. 2006;114(2):242-250.

10. Li Y, Lin J, Yang X, et al. Self-assembled nanoparticles based on amphiphilic anticancer drug-phospholipid complex for targeted drug delivery and intracellular dual-controlled release. ACS Appl Mater Interfaces. 2015;7(32):17573-17581. 
11. Tanhuanpää K, Cheng KH, Anttonen K, Virtanen JA, Somerharju P. Characteristics of pyrene phospholipid/gamma-cyclodextrin complex. Biophys J. 2001;81(3):1501-1510.

12. Patel VF, Liu F, Brown MB. Advances in oral transmucosal drug delivery. J Control Release. 2011;153(2):106-116.

13. Herwadkar A, Banga AK. Peptide and protein transdermal drug delivery. Drug Discov Today. 2012;9(2):e147-e154.

14. Montenegro-Nicolini M, Morales JO. Overview and future potential of buccal mucoadhesive films as drug delivery systems for biologics. AAPS PharmSciTech. 2017;18(1):3-14.

15. Jitendra, Sharma PK, Bansal S, Banik A. Noninvasive routes of proteins and peptides drug delivery. Indian J Pharm Sci. 2011;73(4):367-375.

16. Bhati R. A detailed review on oral mucosal drug delivery system. Int J Pharm Sci Res. 2012;3(3):659-681.

17. Cevc G, Blume G. Lipid vesicles penetrate into intact skin owing to the transdermal osmotic gradients and hydration force. Biochim Biophys Acta. 1992;1104(1):226-232.

18. El Maghraby GM, Williams AC, Barry BW. Oestradiol skin delivery from ultradeformable liposomes: refinement of surfactant concentration. Int J Pharm. 2000;196(1):63-74.

19. Liu L, Zhou C, Xia X, Liu Y. Self-assembled lecithin/chitosan nanoparticles for oral insulin delivery: preparation and functional evaluation. Int J Nanomedicine. 2016;11(1):761.

20. Zeb A, Qureshi OS, Kim HS, Cha JH, Kim HS, Kim JK. Improved skin permeation of methotrexate via nanosized ultradeformable liposomes. Int J Nanomedicine. 2016;11:3813.

21. Amores S, Domenech J, Colom H, et al. An improved cryopreservation method for porcine buccal mucosa in ex vivo drug permeation studies using Franz diffusion cells. Eur J Pharm Sci. 2014;60(1449):49-54.

22. Kim Y, Shields JE. $\mathrm{pH}$ dependent conformational changes in the T- and R-states of insulin in solution: circular dichroic studies in the $\mathrm{pH}$ range of 6 to 10. Biochem Biophys Res Commun. 1992;186(2):1115-1120.

23. Pocker Y, Biswas SB. Conformational dynamics of insulin in solution. Circular dichroic studies. Biochemistry. 1980;19(22):5043-5049.
24. Jin L, Boyd BJ, White PJ, Pennington MW, Norton RS, Nicolazzo JA Buccal mucosal delivery of a potent peptide leads to therapeuticallyrelevant plasma concentrations for the treatment of autoimmune diseases. J Control Release. 2015;199:37-44.

25. Chen R, Li R, Liu Q, et al. Ultradeformable liposomes: a novel vesicular carrier for enhanced transdermal delivery of procyanidins: effect of surfactants on the formation, stability, and transdermal delivery. AAPS PharmSciTech. 2017;18(5):1823-1832.

26. Shelke SS. Phytosomes - a new herbal drug delivery system. Int J Res Pharm Biomed Sci. 2012;3(4):1710-1715.

27. Zana R, Eljebari MJ. Fluorescence probing investigation of the selfassociation of alcohols in aqueous solution. J Phys Chem. 1993;97(42): 11134-11136.

28. Kumar A, Pathak K, Bali V. Ultra-adaptable nanovesicular systems: a carrier for systemic delivery of therapeutic agents. Drug Discov Today. 2012;17(21-22):1233-1241.

29. Cevc G, Schätzlein A, Richardsen H. Ultradeformable lipid vesicles can penetrate the skin and other semi-permeable barriers unfragmented. Evidence from double label CLSM experiments and direct size measurements. Biochim Biophys Acta. 2002;1564(1):21-30.

30. Morrow DIJ, McCarron PA, Woolfson AD, Donnelly RF. Innovative strategies for enhancing topical and transdermal drug delivery. Open Drug Deliv J. 2007;1(1):36-59.

31. Rossi S, Sandri G, Caramella CM. Buccal drug delivery: a challenge already won? Drug Discov Today Technol. 2005;2(1):59-65.

32. Adriaens E, Bytheway H, De Wever B, et al. Successful prevalidation of the slug mucosal irritation test to assess the eye irritation potency of chemicals. Toxicol In Vitro. 2008;22(5):1285-1296.

33. Zaki NM, Mortada ND, Awad GA, Abd Elhady SS. Rapid-onset intranasal delivery of metoclopramide hydrochloride Part II: safety of various absorption enhancers and pharmacokinetic evaluation. Int $J$ Pharm. 2006;327(1-2):97-103.
International Journal of Nanomedicine

\section{Publish your work in this journal}

The International Journal of Nanomedicine is an international, peerreviewed journal focusing on the application of nanotechnology in diagnostics, therapeutics, and drug delivery systems throughout the biomedical field. This journal is indexed on PubMed Central, MedLine, CAS, SciSearch $\AA$, Current Contents $₫ /$ Clinical Medicine,

\section{Dovepress}

Journal Citation Reports/Science Edition, EMBase, Scopus and the Elsevier Bibliographic databases. The manuscript management system is completely online and includes a very quick and fair peer-review system, which is all easy to use. Visit http://www.dovepress.com/ testimonials.php to read real quotes from published authors. 